\title{
Spectral Minutiae Representations of Fingerprints Enhanced by Quality Data
}

\author{
Haiyun Xu and Raymond N.J. Veldhuis
}

\begin{abstract}
Many fingerprint recognition systems are based on minutiae matching. However, the recognition accuracy of minutiae-based matching algorithms is highly dependent on the fingerprint minutiae quality. Therefore, in this paper, we introduce a quality integrated spectral minutiae algorithm, in which the minutiae quality information is incorporated to enhance the performance of the spectral minutiae fingerprint recognition system. In our algorithm, two types of quality data are used. The first one is the minutiae reliability, expressing the probability that a given point is indeed a minutia; the second one is the minutiae location accuracy, quantifying the error on the minutiae location. We integrate these two types of quality information into the spectral minutiae representation algorithm and achieve a decrease in the Equal Error Rate of over $20 \%$ in the experiment.
\end{abstract}

\section{INTRODUCTION}

Recognition of persons by means of biometric characteristics is gaining importance. Among various biometric techniques, such as face, signature and voice, the fingerprint has one of the highest levels of distinctiveness and performance [1] and it is the most commonly used biometric modality. Many fingerprint recognition systems are based on minutiae matching [2], [3]. Minutiae are the endpoints and bifurcations of fingerprint ridges. They are known to remain unchanged over an individual's lifetime and allow a very discriminative classification of fingerprints. The spectral minutiae representation [4] is a novel method to represent a minutiae set as a fixed-length feature vector, which is invariant to translation, and in which rotation and scaling become translations, so that they can be easily compensated for. These characteristics enable the combination of fingerprint recognition systems with template protection schemes ${ }^{1}$ and allow for faster matching as well.

However, the recognition accuracy of minutiae-based matching algorithms is highly dependent on the fingerprint minutiae quality. Reference [9] shows that minutiae-based fingerprint recognition algorithms are less robust to the image quality degradation compared with image-based algorithms. Nowadays, investigating the influence of the fingerprint quality on recognition performances also gains more and more attention [10], [11].

The study presented in [4] shows that the spurious and missing minutiae or/and minutiae location errors can degrade

Haiyun Xu and Raymond N.J. Veldhuis are with Department of Electrical Engineering, University of Twente 7500 AE Enschede, The Netherlands $\{\mathrm{h} . \mathrm{xu}, \mathrm{r.n.j.veldhuis \} @ewi.utwente.nl}$

${ }^{1}$ Our method is designed to use in combination with template protection schemes that are based on fuzzy commitment and helper data schemes, such as [5] and [6], that require as an input a fixed-length feature vector representation of a biometric modality. Other template protection systems exist [7] [8] that do not pose this fixed-length feature vector requirement. the performance of the spectral minutiae recognition system. To cope with low quality fingerprints and to make the spectral minutiae representation algorithm more robust against minutiae errors, we introduce quality integrated spectral minutiae representations of fingerprints, in which the quality information of minutiae is incorporated in the fingerprint representation to enhance the performance of the spectral minutiae fingerprint recognition system.

This paper is organized as follows. First, a review of the spectral minutiae representation is presented in Section II. Next, in Section III, the quality integrated spectral minutiae representations are introduced. Finally, Section IV presents the experimental results and we draw conclusions in Section $\mathrm{V}$.

\section{BACKGROUND}

The spectral minutiae representation is based on the shift, scale and rotation properties of the two-dimensional continuous Fourier transform. In [4], the concept of two representation methods are introduced: the location-based spectral minutiae representation (SML) and the orientationbased spectral minutiae representation (SMO).

\section{A. Spectral Minutiae Representations}

Assume we have a fingerprint with $Z$ minutiae. In SML, with every minutia, a function $m_{i}(x, y)=\delta\left(x-x_{i}, y-\right.$ $\left.y_{i}\right), i=1, \ldots, Z$ is associated where $\left(x_{i}, y_{i}\right)$ represents the location of the $i$-th minutia in the fingerprint image. Thus, in the spatial domain, every minutia is represented by a Dirac pulse. The Fourier transform of $m_{i}(x, y)$ is given by:

$$
\mathscr{F}\left\{m_{i}(x, y)\right\}=\exp \left(-\mathrm{j}\left(\omega_{\mathrm{x}} x_{i}+\omega_{\mathrm{y}} y_{i}\right)\right),
$$

and the location-based spectral minutiae representation is defined as

$$
\mathcal{M}_{\mathrm{L}}\left(\omega_{\mathrm{x}}, \omega_{\mathrm{y}}\right)=\sum_{i=1}^{Z} \exp \left(-\mathrm{j}\left(\omega_{\mathrm{x}} x_{i}+\omega_{\mathrm{y}} y_{i}\right)\right) .
$$

In order to reduce the sensitivity to small variations in minutiae locations in the spatial domain, we use a Gaussian low-pass filter to attenuate the higher frequencies. This multiplication in the frequency domain corresponds to a convolution in the spatial domain where every minutia is now represented by a Gaussian pulse.

Following the shift property of the Fourier transform, the magnitude of $\mathcal{M}$ is taken in order to make the spectrum invariant to translation of the input and we obtain 


$$
\begin{aligned}
& \left|\mathcal{M}_{\mathrm{L}}\left(\omega_{\mathrm{x}}, \omega_{\mathrm{y}} ; \sigma_{\mathrm{L}}^{2}\right)\right|= \\
& \left|\exp \left(-\frac{\omega_{\mathrm{x}}^{2}+\omega_{\mathrm{y}}^{2}}{2 \sigma_{\mathrm{L}}^{-2}}\right) \sum_{i=1}^{Z} \exp \left(-\mathrm{j}\left(\omega_{\mathrm{x}} x_{i}+\omega_{\mathrm{y}} y_{i}\right)\right)\right| .
\end{aligned}
$$

The location-based spectral minutiae representation (SML) only uses the minutiae location information. However, including the minutiae orientation as well may give better discrimination. Therefore, it can be beneficial to also include the orientation information in our spectral representation. The orientation $\theta$ of a minutia can be incorporated by using the spatial derivative of $m(x, y)$ in the direction of the minutia orientation. Thus, to every minutia in a fingerprint, a function $m_{i}(x, y, \theta)$ is assigned being the derivative of $m_{i}(x, y)$ in the direction $\theta_{i}$, such that

$$
\begin{aligned}
& \mathscr{F}\left\{m_{i}(x, y, \theta)\right\}= \\
& \mathrm{j}\left(\omega_{\mathrm{x}} \cos \theta_{i}+\omega_{\mathrm{y}} \sin \theta_{i}\right) \cdot \exp \left(-\mathrm{j}\left(\omega_{\mathrm{x}} x_{i}+\omega_{\mathrm{y}} y_{i}\right)\right) .
\end{aligned}
$$

As with the SML algorithm, using a Gaussian filter and taking the magnitude of the spectrum yields

$$
\begin{aligned}
& \left|\mathcal{M}_{\mathrm{O}}\left(\omega_{\mathrm{x}}, \omega_{\mathrm{y}} ; \sigma_{\mathrm{O}}^{2}\right)\right|=\mid \exp \left(-\frac{\omega_{\mathrm{x}}^{2}+\omega_{\mathrm{y}}^{2}}{2 \sigma_{\mathrm{O}}^{-2}}\right) \\
& \sum_{i=1}^{Z} \mathrm{j}\left(\omega_{\mathrm{x}} \cos \theta_{i}+\omega_{\mathrm{y}} \sin \theta_{i}\right) \cdot \exp \left(-\mathrm{j}\left(\omega_{\mathrm{x}} x_{i}+\omega_{\mathrm{y}} y_{i}\right)\right) \mid \cdot
\end{aligned}
$$

In order to obtain the final spectral representations, the continuous spectra (3) and (5) are sampled on a polarlogarithmic grid. In the radial direction $\lambda$, we use $M=128$ samples between $\lambda_{\mathrm{l}}=0.1$ and $\lambda_{\mathrm{h}}=0.6$. In the angular direction $\beta$, we use $N=256$ samples uniformly distributed between $\beta=0$ and $\beta=\pi$. Because of the symmetry of the Fourier transform for real-valued functions, using the interval between 0 and $\pi$ is sufficient. This polar-logarithmic sampling process is illustrated in Figures 1 and 2. For each spectrum, the horizontal axis represents the rotation angle of the spectral magnitude (from 0 to $\pi$ ); the vertical axis represents the frequency of the spectral magnitude (the frequency increases from top to bottom). The resulting representation in the polar-logarithmic domain is invariant to translation, while rotation and scaling of the input have become translations along the polar-logarithmic coordinates.

\section{B. Spectral Minutiae Matching}

Let $R(m, n)$ and $T(m, n)$ be the two sampled minutiae spectra respectively achieved from the reference fingerprint and test fingerprint. Both $R(m, n)$ and $T(m, n)$ are normalized to have zero mean and unit energy. We use the twodimensional correlation coefficient between $R$ and $T$ as a measure of their similarity.

In practice, the input fingerprint images are rotated and might be scaled (for example, depending on the sensor that is used to acquire an image). Assume that the scaling has already been compensated for on the level of the minutiae

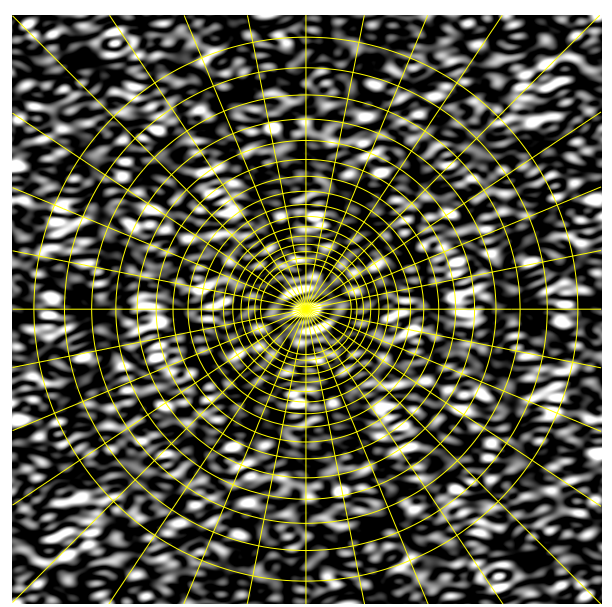

(a)

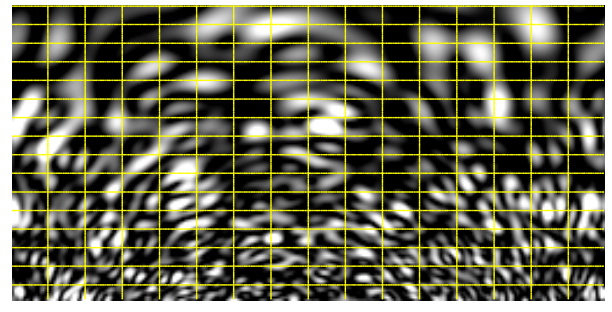

(b)

Fig. 1. Illustration of the polar-logarithmic sampling (SML spectra). (a) the Fourier spectrum in a Cartesian coordinate and a polar-logarithmic sampling grid; (b) the Fourier spectrum sampled on a polar-logarithmic grid.

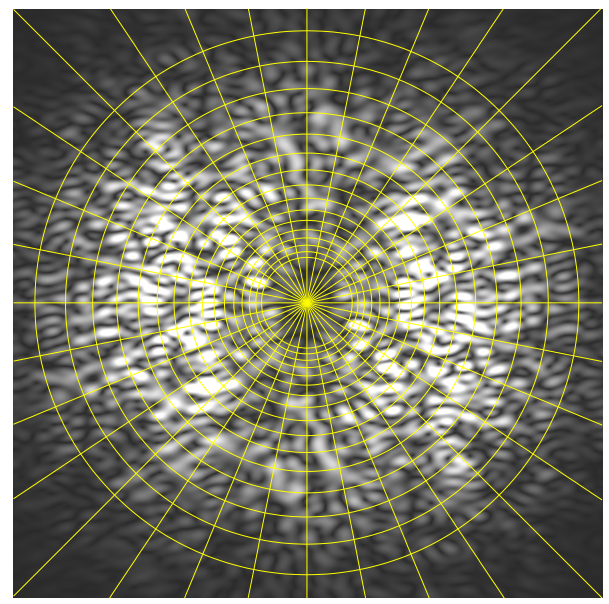

(a)

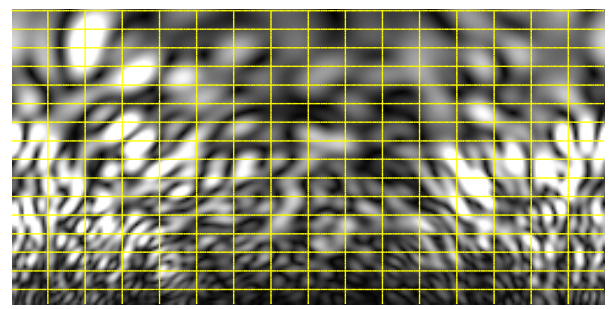

(b)

Fig. 2. Illustration of the polar-logarithmic sampling (SMO spectra). (a) the Fourier spectrum in a Cartesian coordinate and a polar-logarithmic sampling grid; (b) the Fourier spectrum sampled on a polar-logarithmic grid. 
sets [12]. Then we only need to test a few rotations, which become the circular shifts in the horizontal direction. We denote $T\left(m, n-n_{c s}\right)$ as a circular shifted version of $T(m, n)$. We use the fast rotation shift searching algorithm that was presented in $[13]^{2}$ and choose the maximum score of the different combinations as the final matching score between $R$ and $T$,

$$
\begin{array}{r}
S^{(R, T)}=\max _{n_{c s}}\left\{\frac{1}{M N} \sum_{m, n} R(m, n) T\left(m, n-n_{c s}\right)\right\} \\
-15 \leq n_{c s} \leq 15 .
\end{array}
$$

\section{Quality Integrated Spectral Minutiae REPRESENTATIONS}

In order to improve the performance of the spectral minutiae representation, we incorporate minutiae quality data in the calculation of the spectral minutiae. Minutiae quality is described by two numbers. The first one is the minutiae reliability (QM), expressing the probability that a given point is indeed a minutia. This measure is recorded following the specification of the minutiae template standard ISO/IEC 19794-2 [12]: the quality figure ranges from 100 as a maximum to 1 as a minimum. The other quality data we incorporated is the minutiae location accuracy $(\mathrm{QL})$, quantifying the error on the minutiae location. This measure is defined as the radius, in pixels, of a circle from the found minutiae position, within which the minutiae position is located. It is provided by a proprietary algorithm.

\section{A. Using Quality of Minutiae Reliability (QM)}

The quality of minutiae reliability (QM) gives the certainty that a given point is indeed a minutia. We use it in the spectral minutiae representation by weighing the Dirac pulse assigned to each minutia. For each minutia, the weight $w$ depends linearly on the minutiae reliability quality $q_{\mathrm{M}}$. A higher $q_{\mathrm{M}}$ (which means a minutia with higher reliability) corresponds to a higher weight $w$. Then, SML in (3) becomes

$$
\begin{aligned}
& \left|\mathcal{M}_{\mathrm{L}}\left(\omega_{\mathrm{x}}, \omega_{\mathrm{y}} ; \sigma_{\mathrm{L}}^{2}\right)\right|= \\
& \left|\exp \left(-\frac{\omega_{\mathrm{x}}^{2}+\omega_{\mathrm{y}}^{2}}{2 \sigma_{\mathrm{L}}^{-2}}\right) \sum_{i=1}^{Z} w_{i} \exp \left(-\mathrm{j}\left(\omega_{\mathrm{x}} x_{i}+\omega_{\mathrm{y}} y_{i}\right)\right)\right|,
\end{aligned}
$$

and SMO in (5) becomes

$$
\begin{aligned}
& \left|\mathcal{M}_{\mathrm{O}}\left(\omega_{\mathrm{x}}, \omega_{\mathrm{y}} ; \sigma_{\mathrm{O}}^{2}\right)\right|=\mid \exp \left(-\frac{\omega_{\mathrm{x}}^{2}+\omega_{\mathrm{y}}^{2}}{2 \sigma_{\mathrm{O}}^{-2}}\right) \\
& \sum_{i=1}^{Z} \mathrm{j}\left(\omega_{\mathrm{x}} \cos \theta_{i}+\omega_{\mathrm{y}} \sin \theta_{i}\right) \cdot w_{i} \exp \left(-\mathrm{j}\left(\omega_{\mathrm{x}} x_{i}+\omega_{\mathrm{y}} y_{i}\right)\right) \mid .
\end{aligned}
$$

Equations (7) and (8) are the expressions of the minutiae reliability incorporated SML and SMO.

\footnotetext{
${ }^{2}$ In [13], totaly 9 rotations are tested in a range of $-10^{\circ}$ to $+10^{\circ}$ in case of $N=256$ samples between 0 to $\pi$.
}

\section{B. Using Quality of Minutiae Location Accuracy (QL)}

The quality of minutiae location accuracy (QL) quantifies the error on the minutiae location. As we mentioned in Section II, we use a Gaussian low-pass filter to attenuate the higher frequencies in the minutiae spectrum in order to reduce the sensitivity to the minutiae location errors in the spatial domain. Therefore, we use this minutiae location quality measure to adjust the Gaussian parameters $\sigma_{\mathrm{L}}$ and $\sigma_{\mathrm{O}}$ in the spectral minutiae representations. For each minutia, the Gaussian parameter $\sigma$ depends linearly on the minutiae location accuracy $q_{\mathrm{L}}$. A higher $q_{\mathrm{L}}$ (which means a minutia with lower location accuracy) corresponds to a higher $\sigma$. Then, SML in (3) becomes

$$
\begin{aligned}
& \left|\mathcal{M}_{\mathrm{L}}\left(\omega_{\mathrm{x}}, \omega_{\mathrm{y}}\right)\right|= \\
& \left|\sum_{i=1}^{Z} \exp \left(-\frac{\omega_{\mathrm{x}}^{2}+\omega_{\mathrm{y}}^{2}}{2 \sigma_{\mathrm{L} i}^{-2}}\right) \cdot \exp \left(-\mathrm{j}\left(\omega_{\mathrm{x}} x_{i}+\omega_{\mathrm{y}} y_{i}\right)\right)\right|,
\end{aligned}
$$

and SMO in (5) becomes

$$
\begin{aligned}
& \left|\mathcal{M}_{\mathrm{O}}\left(\omega_{\mathrm{x}}, \omega_{\mathrm{y}}\right)\right|=\mid \sum_{i=1}^{Z} \exp \left(-\frac{\omega_{\mathrm{x}}^{2}+\omega_{\mathrm{y}}^{2}}{2 \sigma_{\mathrm{O} i}^{-2}}\right) \\
& \mathrm{j}\left(\omega_{\mathrm{x}} \cos \theta_{i}+\omega_{\mathrm{y}} \sin \theta_{i}\right) \cdot \exp \left(-\mathrm{j}\left(\omega_{\mathrm{x}} x_{i}+\omega_{\mathrm{y}} y_{i}\right)\right) \mid .
\end{aligned}
$$

Equations (9) and (10) are the expressions of the minutiae location accuracy incorporated SML and SMO.

\section{Using both $Q M$ and $Q L$}

If we incorporate both $\mathrm{QM}$ and $\mathrm{QL}$ following the algorithms presented in III-A and III-B, we obtain SML in (3) as

$$
\begin{aligned}
& \left|\mathcal{M}_{\mathrm{L}}\left(\omega_{\mathrm{x}}, \omega_{\mathrm{y}}\right)\right|= \\
& \left|\sum_{i=1}^{Z} \exp \left(-\frac{\omega_{\mathrm{x}}^{2}+\omega_{\mathrm{y}}^{2}}{2 \sigma_{\mathrm{L} i}^{-2}}\right) \cdot w_{i} \exp \left(-\mathrm{j}\left(\omega_{\mathrm{x}} x_{i}+\omega_{\mathrm{y}} y_{i}\right)\right)\right|,
\end{aligned}
$$

and SMO in (5) as

$$
\begin{aligned}
& \left|\mathcal{M}_{\mathrm{O}}\left(\omega_{\mathrm{x}}, \omega_{\mathrm{y}}\right)\right|=\mid \sum_{i=1}^{Z} \exp \left(-\frac{\omega_{\mathrm{x}}^{2}+\omega_{\mathrm{y}}^{2}}{2 \sigma_{\mathrm{O} i}^{-2}}\right) \\
& \mathrm{j}\left(\omega_{\mathrm{x}} \cos \theta_{i}+\omega_{\mathrm{y}} \sin \theta_{i}\right) \cdot w_{i} \exp \left(-\mathrm{j}\left(\omega_{\mathrm{x}} x_{i}+\omega_{\mathrm{y}} y_{i}\right)\right) \mid .
\end{aligned}
$$

Equations (11) and (12) are the expressions of the quality integrated SML and SMO.

\section{EXPERIMENTS}

We test the quality integrated spectral minutiae representations (Equations (7) to (12)) in a verification setting. The matching performance of a fingerprint verification system can be evaluated by the false acceptance rate (FAR), the false rejection rate (FRR), and the equal error rate (EER). 


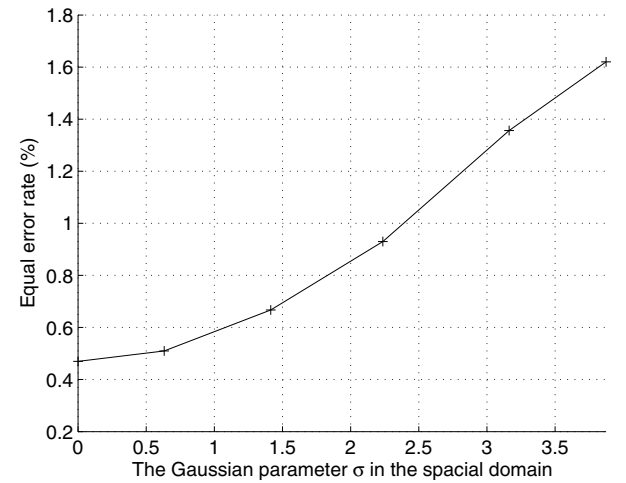

Fig. 3. Relationship of the Gaussian parameter $\sigma$ (in the spacial domain) and SML performances (MCYT VeriFinger minutiae set in [4]).

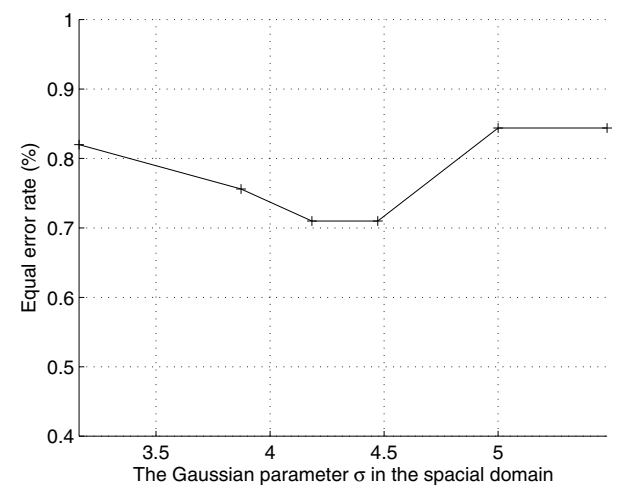

Fig. 4. Relationship of the Gaussian parameter $\sigma$ (in the spacial domain) and SMO performances (MCYT VeriFinger minutiae set in [4]).

When the decision threshold of a biometric security system is set such that the FAR and FRR are equal, the common value of FAR and FRR is referred to as the EER. In this paper, we use FAR, EER and the genuine accept rate (GAR), $\mathrm{GAR}=1-\mathrm{FRR}$, as performance indicators of our scheme.

The proposed algorithms are evaluated on the FVC2002DB2 [14] fingerprint database. The minutiae sets including the minutiae quality data are extracted by a proprietary method. The experiment is implemented following the experimental setting and test protocol in $[4]^{3}$. A correlation based matching algorithm is used and a score level sum rule for SML and SMO is applied.

From our experiments, we noticed that for SML and SMO, we need to choose different Gaussian parameters $\left(\sigma_{\mathrm{L}}\right.$ and $\sigma_{\mathrm{O}}$ ) to achieve the best performances. Figures 3 and 4 show the influence of the Gaussian parameter $\sigma$ to the performances of SML and $\mathrm{SMO}^{4}$. We noticed that the Gaussian parameter has larger effects on SML than on SMO. Moreover, a Gaussian kernel is needed for SMO for achieving a better performance, while for SML it is

\footnotetext{
${ }^{3}$ In [4], for each fingerprint, maximum two cores or/and two deltas were used to improve the performance. In this paper, only the upper core is used as a reference point to enhance the recognition accuracy.

${ }^{4}$ Figures 3 and 4 are acquired by the experiments on the MCYT fingerprint database in [4]. The influence of the Gaussian parameter $\sigma$ to the SML and SMO performances is similar for different fingerprint databases.
}

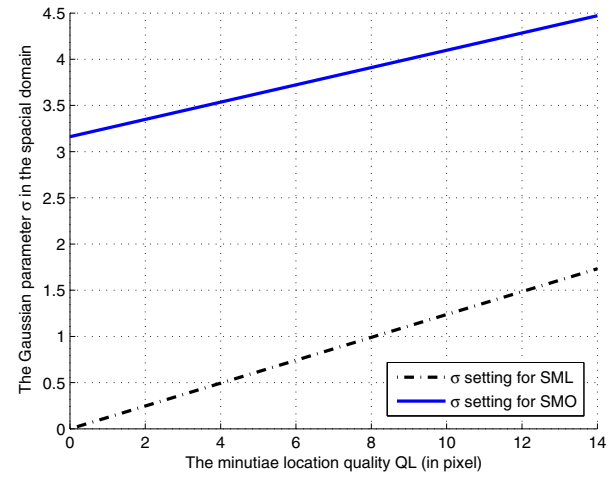

Fig. 5. Relationship of the Gaussian parameter $\sigma$ and the minutiae location quality QL.

not. The reason is that because the minutiae orientation is incorporated as a derivative of the delta function (see Equation (4)), this will amplify the noise (both in minutiae location and orientation) in the high frequency part in SMO. Therefore, a Gaussian kernel is needed for SMO to attenuate the higher frequencies. In SML, the high frequency part contains discriminative information, while the noise is evenly distributed in all frequencies, therefore, a Gaussian kernel does not help for a better performance. In our experiments, when no minutiae location quality is incorporated, we chose $\sigma=0$ for SML (in this case, no multiplication with Gaussian in the frequency domain) and $\sigma=4.24$ for SMO. In case the fingerprint resolution is 569dpi (the FVC2002DB2 database), the Gaussian parameter $\sigma=4.24$ (pixel) in the spacial domain is about $0.19(\mathrm{~mm})$ in reality. When incorporating the minutiae location accuracy (QL), we use the linear relationships shown in Figure 5 to adjust the Gaussian parameter $\sigma$ according to the minutiae location quality.

The final recognition performances are shown in Table I and the ROC curves are shown in Figure 6. From the results, we can see that the recognition performance of the spectral minutiae representation improves after incorporating the minutiae reliability quality $(\mathrm{QM})$. However, the improvement by using minutiae location accuracy (QL) is very limited. This may result from the low reliability of the minutiae location accuracy quality data. In Figure 7 , we further illustrate the genuine and imposter matching score densities of different cases. From the matching score densities, we can notice that after incorporating the quality data, the imposter score densities are almost keep the same, while the genuine scores are increased. This also explains the enhancements in recognition accuracy after integrating the quality data into the spectral minutiae representations. By incorporating both quality data (QM and QL), we achieve a decrease of more than $20 \%$ in equal error rate in the experiment.

\section{CONCLUSIONS}

In fingerprint recognition systems, low quality fingerprints are unavoidable. To make the spectral minutiae represen- 
TABLE I

RESULTS ON THE FVC2002-DB2 DATABASE.

\begin{tabular}{|l|c|c|c|c|}
\hline \multirow{2}{*}{ Methods } & \multirow{2}{*}{ EER } & \multicolumn{3}{|c|}{ GAR } \\
\cline { 3 - 5 } & & FAR $=1 \%$ & FAR $=0.1 \%$ & FAR $=0 \%$ \\
\hline No quality & $4.5 \%$ & $94.2 \%$ & $91.7 \%$ & $88.8 \%$ \\
\hline QM & $3.7 \%$ & $95.2 \%$ & $93.5 \%$ & $90.5 \%$ \\
\hline QL & $4.0 \%$ & $94.3 \%$ & $92.0 \%$ & $89.0 \%$ \\
\hline QM \& QL & $3.5 \%$ & $95.2 \%$ & $93.2 \%$ & $91.0 \%$ \\
\hline
\end{tabular}

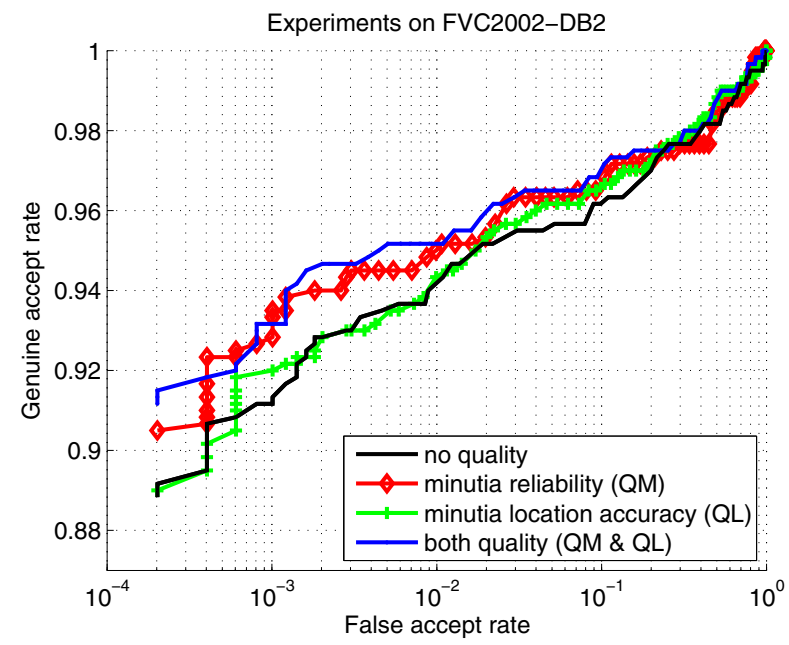

Fig. 6. ROC curves on FVC2002-DB2 for different cases.

tation system more robust against minutiae errors, we incorporate minutiae quality in the calculation of the spectral minutiae representation. In this paper, we introduce two methods to incorporate minutiae reliability (QM) and minutiae location accuracy (QL) respectively. The experiments show that the performance of the spectral minutiae representation can be improved by using the minutiae quality data. The QM incorporated spectral minutiae representation shows better results than the QL incorporated spectral minutiae representation. By using both quality data, we achieve overall the best result.

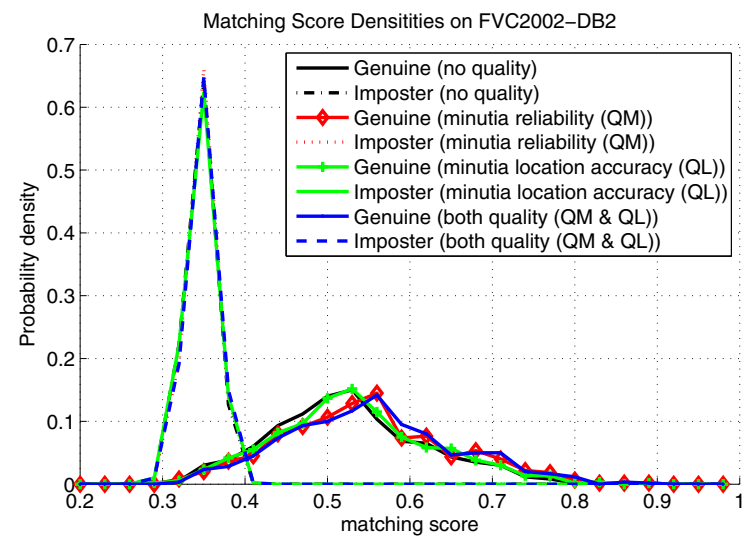

Fig. 7. Matching score densities on FVC2002-DB2 for different cases.
This paper presents the advantage of incorporating quality data in the spectral minutiae representation system. The proposed methods only vary the minutiae representations, while keeping the matching algorithm unchanged, so that they can be easily integrated in the spectral minutiae recognition system. Our future work will be the interoperability study of using quality data and algorithm optimization of incorporating the minutiae quality data to enhance the recognition performance.

\section{ACKNOWLEDGMENTS}

This research is supported by the ProBiTe project funding by Sentinels and the TURBINE project funding by the European Union under the Seventh Framework Programme.

\section{REFERENCES}

[1] D. Maltoni, D. Maio, A.K. Jain, and S. Prabhakar. Handbook of Fingerprint Recognition. Springer, New York, 2003.

[2] A.K. Jain, L. Hong, and R. Bolle. On-line fingerprint verification. IEEE Trans. PAMI, 19(4):302-314, April 1997.

[3] VeriFinger SDK. http://www.neurotechnologija.com/.

[4] H. Xu, R. N. J. Veldhuis, A. M. Bazen, T. A. M. Kevenaar, and A. H. M. Akkermans. Fingerprint verification using spectral minutiae representations. IEEE Transactions on Information Forensics and Security. To appear.

[5] P. Tuyls, A.H.M. Akkermans, T.A.M. Kevenaar, G.J. Schrijen, A.M. Bazen, and R.N.J. Veldhuis. Practical biometric authentication with template protection. In AVBPA, pages 436-446, 2005.

[6] Ari Juels. Fuzzy commitment. In Pim Tuyls, Boris Skoric, and Tom Kevenaar, editors, Security with Noisy Data - On Private Biometrics, Secure Key Storage and Anti-Counterfeiting, chapter 3, pages 45-56. Springer, 2007.

[7] K. Nandakumar, A.K. Jain, and S. Pankanti. Fingerprint-based fuzzy vault: Implementation and performance. Information Forensics and Security, IEEE Transactions on, 2(4):744-757, Dec. 2007.

[8] T.E. Boult, W.J. Schdrer, and R. Woodworth. Revocable fingerprint biotokens: Accuracy and security analysis. In Computer Vision and Pattern Recognition, 2007. CVPR '07. IEEE Conference on, pages 1-8, June 2007.

[9] Julian Fierrez-aguilar, Javier Ortega-garcia, and Anil K. Jain. Incorporating image quality in multi-algorithm fingerprint verification. In Proc. IAPR Intl. Conf. on Biometrics, ICB, Springer LNCS-3832, pages 213-220. Springer, 2006.

[10] Sanghoon Lee, Heeseung Choi, Kyoungtaek Choi, and Jaihie Kim. Fingerprint-quality index using gradient components. Information Forensics and Security, IEEE Transactions on, 3(4):792-800, Dec. 2008.

[11] H. Fronthaler, K. Kollreider, J. Bigun, J. Fierrez, F. Alonso-Fernandez, J. Ortega-Garcia, and J. Gonzalez-Rodriguez. Fingerprint imagequality estimation and its application to multialgorithm verification. Information Forensics and Security, IEEE Transactions on, 3(2):331338, June 2008.

[12] ISO/IEC 19794-2, Information Technology - Biometric Data Interchange Format - Part 2: Finger Minutiae Data. 2005.

[13] H. Xu, R. N. J. Veldhuis, T. A. M. Kevenaar, A. H. M. Akkermans, and A. M. Bazen. Spectral Minutiae: A Fixed-length Representation of a Minutiae Set. In Proceedings of the IEEE Computer Society Conference on Computer Vision and Pattern Recognition - Workshop on Biometrics, Anchorage, USA, 2008.

[14] D. Maio, D. Maltoni, R. Cappelli, J.L. Wayman, and A.K. Jain. FVC2002: Second fingerprint verification competition. 3:811-814, August 2002. 